\title{
Berufsrechtsnovelle
}

\section{Kooperationen werden leichter}

\author{
Mit der Novelle der Musterberufsordnung erhalten vor allem Teilbe- \\ rufsausübungsgemeinschaften mehr Spielraum bei der Kooperation, \\ ohne gleich unter den Verdacht verbotener Zuweisungen zu fallen.
}

A $\mathrm{n}$ vier Paragrafen der Musterberufsordnung (MBO) hat der 118. Deutsche Ärztetag geschraubt. Seit Juli ist die zugehörige Novelle der $\mathrm{MBO}$ auch in Kraft.

$\$ 15$ Abs. 3 MBO: Für die Forschung am Menschen müssen Ärzte die überarbeitete Fassung der Deklaration von Helsinki des Weltärztebundes aus 2013 beachten. Diese spezifiziert zum Beispiel die Anforderungen für Maßnahmen nach Abschluss einer Studie.

\$2 20 Abs. 2 MBO: Verstirbt der Praxisinhaber, kann die Praxis zugunsten seiner Witwe oder einer/m nach dem Gesetz eingetragenen Lebenspartner/in durch einen anderen Arzte weitergeführt werden. Und zwar für sechs Monate.

Dabei ist vor allem die Änderung der pauschalen Kriminalisierung von Teilberufsausübungsgemeinschaften erst durch das Eingreifen des Bundesgerichtshofs (BGH) zustande gekommen. Dagegen ging die Anpassung ans Patientenrechtegesetz aus dem Jahr 2013 verhältnismäßig zügig vonstatten. In einem langen Streit zwischen der Wettbewerbszentrale Bad Homburg und einer badenwürttembergischen Teilberufsausübungsgemeinschaft (TBAG) stellte das Landgericht Mosbach bereits 2011 fest, dass Paragraf 18 Absatz 1 der Berufsordnung für Ärzte gegen das Grundgesetz verstößt. In dem Paragrafen, den die baden-württembergische Ärztekammer aus der MBO übernommen hatte, stand bis vor Kurzem, dass bei einer TBAG, in der sich ein oder mehrere Ärzte auf das Erbringen medizinisch-technischer Leistungen auf Veranlassung der übrigen Mitglieder der TBAG beschränken, generell von einer Umgehung des Zuweisungsverbots nach Paragraf $31 \mathrm{MBO}$ ausgegangen wird. Nach Ansicht des Landgerichts Mosbach (Az.: 3 $\mathrm{O} 13 / 10$ ) ist das eine klare berufliche Benachteiligung von Radiologen, deren Betätigungsfeld nun einmal gerade solche Leistungen und dies in der Regel auf Veranlassung anderer Ärzte seien. Der Fall landete schließlich beim Bundesgerichtshof hoch, der 2014 entschied: Die Regelung in der $\mathrm{MBO}$ ist verfassungswidrig.

Im Prinzip steht Ärzten schon seit Mai 2014, als das Urteil des BGH gefallen ist (Az.: I ZR 137/12), der Weg zu einer Kooperation mit Radiologen, Nuklearmedizinern und weiteren Fachkollegen, die rein medizinisch-technische Leistungen erbringen, offen. Allerdings müssen sie dabei auf eine leistungsgerechte Gewinnverteilung achten. Mit der Änderung von Paragraf 18 Absatz 1 der MBO sind sie aber auch weitgehend vor erneuten Rechtsstreitereien gefeit. Denn dort steht nunmehr nur noch: Eine Umgehung des Zuweisungsverbots ,liegt insbesondere dann vor, wenn der Gewinn ohne Grund in einer Weise verteilt wird, die nicht dem Anteil der persönlich erbrachten Leistungen entspricht". Es ist sehr wahrscheinlich, dass die Landesärztekammern die Änderung ebenfalls in ihre regional gültigen Berufsordnungen aufnehmen werden. Das bedeute jedoch nicht, dass die
Kammer Zuweisungen in Teil-BAG künftig nicht mehr prüfen werde, sagteVolker Heiliger, Pressesprecher der Ärztekammer Westfalen-Lippe. Bis die Berufsordnungen auf Landesebene angepasst sind, dauert es. In Hessen findet die Kammerversammlung immerhin schon im September statt, damit könnte die novellierte Berufsordnung im November in Kraft treten. In Westfalen-Lippe, ebenso wie in Mecklenburg-Vorpommern und Niedersachsen wird sich die Kammerversammlung erst im November mit den Änderungen in der MBO befassen.

Dabei wurde auch an anderer Stelle nachgebessert: Paragraf 10 Absatz 2 der MBO gibt Patienten nun mehr Rechte zur Einsicht in die Dokumentation der Ärzte. Der Ärztetag hat hier bewusst den Begriff "Dokumentation" verwendet, weil die reine Patientenakte nicht ,alle relevanten Konstellationen ärztlicher Dokumentation erfasst". Gestrichen wurde der Passus, der die subjektiven Notizen des Arztes vom Einsichtsrecht ausgeschlossen hatte. Damit folgt der Ärztetag dem geltenden Patientenrechtegesetz und der Rechtsprechung des Bundesverfassungsgerichts. Das hatte schon vor Inkrafttreten des $\mathrm{Pa}$ tientenrechtegesetz festgestellt (9/1998: Az.: 1 BvR 1130/98; 1/2006: Az.: 2 BvR 443/02), dass eine pauschale Beschränkung des Einsichtnahmerechts der Patienten auf objektive Befunde und Aufzeichnungen in der ärztlichen Dokumentation nicht verfassungskonform ist. Nach der MBO-Novelle ist das Einsichtsrecht des Patienten nur dann beschränkt, wenn erhebliche therapeutische Gründe oder Rechte des Arztes oder Dritter dem entgegenstehen. Solch eine Einschränkung sei aber auf das notwendige Minimum zu beschränken. Rebekka Höhl 